\title{
ON CHARACTERIZATIONS OF FIXED POINTS
}

\author{
ZEQING LIU, LILI ZHANG, and SHIN MIN KANG
}

(Received 16 January 2001 and in revised form 14 March 2001)

\begin{abstract}
We give some necessary and sufficient conditions for the existence of fixed points of a family of self mappings of a metric space and we establish an equivalent condition for the existence of fixed points of a continuous compact mapping of a metric space.
\end{abstract}

2000 Mathematics Subject Classification. 54H25.

1. Introduction. Jungck [2] first gave a necessary and sufficient condition for the existence of fixed points of a continuous self mapping of a complete metric space. Afterwards, Park [8], Leader [4], and Khan and Fisher [3] established a few theorems similar to that of Jungck. Janos [1] and Park [9] proved fixed point theorems for compact self mappings of a metric space. Recently, Liu [5] established criteria for the existence of fixed points of a family of self mappings of a metric space. The aim of this paper is to offer some characterizations for the existence of fixed points of a family of self mappings and a continuous compact mappings of metric spaces, respectively. We also establish a fixed point theorem for two compact mappings, which extends properly the results of Janos [1] and Park [9].

Let $\omega$ and $N$ denote the sets of nonnegative and positive integers, respectively. Suppose that $(X, d)$ is a metric space. For $x, y \in X$, define

$$
\begin{aligned}
C_{f} & =\{g \mid g: X \longrightarrow X \text { and } f g=g f\}, \\
H_{f} & =\left\{g \mid g: X \longrightarrow X \text { and } g \cap_{n \in \omega} f^{n} X \subseteq \cap_{n \in \omega} f^{n} X\right\}, \\
H_{f}(x) & =\left\{h x \mid h \in H_{f}\right\}, \quad H_{f}(x, y)=H_{f}(x) \cup H_{f}(y), \\
O(x, f) & =\left\{f^{n} x \mid n \in \omega\right\}, \quad O(x, y, f)=O(x, f) \cup O(y, f) .
\end{aligned}
$$

Obviously, $C_{f} \subseteq H_{f}$. Let $\Phi$ be a family of self mappings of $X$. A point $x \in X$ is said to be a fixed point of $\Phi$ if $f x=x$ for all $f \in \Phi$. Let $F: X \times X \rightarrow[0,+\infty)$ be continuous and $F(x, y)=0$ if and only if $x=y$. For $A, B \subset X$, define

$$
\delta(A, B)=\sup \{F(x, y) \mid x \in A, y \in B\}
$$

and $\delta(A)=\delta(A, A)$. Particularly, $d(A)=\sup \{d(x, y) \mid x, y \in A\}$. Let $M(X)$ denote the set of all metrics on $X$ that are topologically equivalent to $d$ for a given metric space $(X, d)$. A self mapping $f$ of a metric space $(X, d)$ is said to be compact if there exists a compact set $Y$ satisfying $f X \subseteq Y \subseteq X$. 
In order to prove our main results, we need the following lemmas.

LEMMA 1.1 (see [6]). Let $f$ be a continuous compact self mapping of a metric space $(X, d)$. If $A=\cap_{n \in \omega} f^{n} X$, then
(a1) $A$ is compact,
(a2) $f A=A \neq \varnothing$,
(a3) $d\left(f^{n} X\right) \rightarrow d(A)$ as $n \rightarrow \infty$,
(a4) $\left\{f^{n} \mid n \in \omega\right\} \subseteq H_{f}$.

LEMMA 1.2 (see [7]). Let $f$ be a continuous self mappings of a metric space $(X, d)$ with the following properties:

(a5) $f$ has a unique fixed point $w$ in $X$,

(a6) for every $x \in X$, the sequence of iterations $\left\{f^{n} x\right\}_{n=0}^{\infty}$ converges to $w$,

(a7) there exists an open neighborhood $U$ of $w$ with the property that given any open set $V$ containing $w$, there exists $k \in N$ such that $n \geq k$ implies $f^{n} U \subset V$.

Then for each $\alpha \in(0,1)$, there exists a metric $d^{\prime} \in M(X)$ relative to which $f$ is a contraction with Lipschitz constant $\alpha$.

\section{Main results}

THEOREM 2.1. Let $\Phi$ be a family of self mappings of a metric space $(X, d)$. Then the following statements are equivalent:

(b1) $\Phi$ has a fixed point;

(b2) there exist $m, n \in N$ and continuous compact self mappings $f, g$ of $(X, d)$ such that either $\Phi \subseteq C_{f}$ or $\Phi \subseteq C_{g}$ and

$$
F\left(f^{m} x, g^{n} y\right)<\delta\left(H_{f}(x), H_{g}(y)\right),
$$

for all $x, y \in X$ with $f^{m} x \neq g^{n} y$;

(b3) there exist $m, n \in N$ and continuous self mappings $f, g$ of $(X, d)$ such that $f g$ is compact, $f \in C_{g}, \Phi \in C_{f g}$, and

$$
F\left(f^{m} x, g^{n} y\right)<\delta\left(H_{f g}(x, y)\right)
$$

for all $x, y \in X$ with $f^{m} x \neq g^{n} y$,

(b4) there exists a continuous compact self mapping of $(X, d)$ with $\Phi \subseteq C_{f}$ such that

$$
\begin{gathered}
F(f x, f y)<\max \left\{F(x, y), F(x, f x), F(y, f y), \frac{F(x, f x) F(y, f y)}{F(x, y)},\right. \\
\left.\frac{F(f x, f y) F(x, f x)}{F(x, y)}, \frac{F(x, f y) F(f x, y)}{F(x, y)}\right\},
\end{gathered}
$$

for all $x, y \in X$ with $x \neq y$.

Moreover, if (b2) holds, then $f, g$, and $\Phi$ have a unique common fixed point; if (b3) holds, then $f g$ and $\Phi$ have a unique common fixed point; if (b4) holds, then $f$ and $\Phi$ have a unique common fixed point. 
Proof. Let (b1) hold and $w$ be a fixed point of $\Phi$. Define $f, g: X \rightarrow X$ by $f x=g x=$ $w$ for all $x \in X$. It is easy to show that (b2), (b3), and (b4) hold.

Assume that (b2) holds. Let $A=\cap_{n \in \omega} f^{n} X, B=\cap_{n \in \omega} g^{n} X$. Since $f$ and $g$ are continuous compact self mappings of $(X, d)$, it follows from (a1) and (a2) that $A$ and $B$ are compact and $f A=A, g B=B$. Consequently, $f^{m} A=A, g^{n} B=B$. Suppose that $\delta(A, B)>0$. Then there exist $a \in A, b \in B$ with $\delta(A, B)=F(a, b)$ because $F$ is continuous and $A \times B$ is compact. Since $f^{m} A=A, g^{n} B=B$, there exist $x \in A, y \in B$ such that $f^{m} x=a, g^{n} y=b$. In view of (2.1), we have

$$
\delta(A, B)=F(a, b)=F\left(f^{m} x, g^{n} y\right)<\delta\left(H_{f}(x), H_{g}(y)\right)<\delta(A, B),
$$

which is impossible, and hence $\delta(A, B)=0$. That is, $A=B=\{w\}$ for some $w \in X$ so $f w=g w=w$. If $v$ is another fixed point of $f$, then $v \in \cap_{n \in \omega} f^{n} X=\{w\}$, that is, $v=w$. Hence $w$ is the only fixed point of $f$. Similarly, $w$ is also the only fixed point of $g$.

Without loss of generality, we assume that $\Phi \subseteq C_{f}$. It follows from $C_{f} \subseteq H_{f}$ that $h A \subseteq A$ for all $h \in \Phi$. That is, $h w=w$ for all $h \in \Phi$. Thus $w$ is the only common fixed point of $f, g$, and $\Phi$. Therefore (b1) holds.

Assume that (b3) holds. Put $A=\cap_{n \in \omega}(f g)^{n} X$. Then $A$ is compact and $f g A=A$. Since $f$ is continuous and $f \in C_{g}$, we infer that

$$
f A=f \cap_{n \in \omega}(f g)^{n} X \subseteq \cap_{n \in \omega}(f g)^{n} f X \subseteq \cap_{n \in \omega}(f g)^{n} X=A .
$$

Similarly, we have

$$
g A \subseteq A
$$

It follows from $f g A=A$, (2.5), and (2.6) that

$$
f A \subseteq A=f g A \subseteq f A .
$$

That is, $f A=A$. Similarly, we have $g A=A$. Suppose that $\delta(A)>0$. Because $F$ is continuous and $A$ is compact, then there exist $a, b \in A$ such that $\delta(A)=F(a, b)$. Since $f^{m} A=g^{n} A=A$, there exist $x, y \in A$ with $f^{m} x=a, g^{n} y=b$. Using (2.2), we have

$$
\delta(A)=F(a, b)=\left(f^{m} x, g^{n} y\right)<\delta\left(H_{F G}(x, y)\right) \leq \delta(A),
$$

which is a contradiction. Hence $\delta(A)=0$. That is, $A=\{w\}$ for some $w \in X$. This implies that $f w=g w=f g w=w$. As in the proof of above, we can prove that $w$ is the only fixed point of $f g$, and $w$ is the unique common fixed point of $f g$ and $\Phi$. So (b1) holds.

Assume that (b4) holds. As above we infer that $A=\cap_{n \in \omega} f^{n} X$ is compact and $f A=$ $A$. Since $F$ is continuous, the function $\phi(x)$ defined by $\phi(x)=F(x, f x)$ for $x \in A$ is continuous and attains its minimum value at some $w \in A$. Suppose that $w \neq f w$. By 
virtue of (2.3), we get

$$
\begin{aligned}
\phi(f w)= & F(f w, f f w) \\
< & \max \left\{F(w, f w), F(w, f w), F(f w, f f w), \frac{F(w, f w) F(f w, f f w)}{F(w, f w)},\right. \\
& \left.\quad \frac{F(f w, f f w) F(w, f w)}{F(w, f w)}, \frac{F(w, f f w) F(f w, f w)}{F(w, f w)}\right\} \\
= & F(w, f w) \\
= & \phi(w) .
\end{aligned}
$$

This is a contradiction to the definition of $w$. So $w$ is a fixed point of $f$. If $f$ has a second distinct fixed point $v$, by (2.3), we obtain that

$$
\begin{aligned}
F(w, v)= & F(f w, f v) \\
<\max \left\{F(w, v), F(w, w), F(v, v), \frac{F(w, w) F(v, v)}{F(w, v)},\right. & \left.\frac{F(w, v) F(w, w)}{F(w, v)}, \frac{F(w, v) F(w, v)}{F(w, v)}\right\} \\
= & F(w, v),
\end{aligned}
$$

which is a contradiction. Therefore, $w$ is the only fixed point of $f$. It is a simple matter to show that $w$ is the unique common fixed point of $f$ and $\Phi$. Thus (b1) holds. This completes the proof.

Next, we give a theorem about the equivalent condition for the existence of fixed points of a continuous compact self mapping on a metric space.

THEOREM 2.2. Let $s$ be a continuous compact self mapping of a metric space $(X, d)$. Then $s$ has a fixed point if and only if there exists a continuous self mapping $f$ of $X$ such that $f \in C_{s}$ and

$$
\begin{gathered}
F(f x, f y)<\max \left\{F(s x, s y), F(s x, f x), F(s y, f y), \frac{F(s x, f x) F(s y, f y)}{F(s x, s y)},\right. \\
\left.\frac{F(f x, f y) F(s x, f x)}{F(s x, s y)}, \frac{F(s x, f y) F(f x, s y)}{F(s x, s y)}\right\},
\end{gathered}
$$

for all $x, y \in X$ with $s x \neq s y$. Indeed, $f$ and $s$ have a unique common fixed point.

Proof. To see that the stated conditions is necessary, suppose that $s$ has a fixed point $w \in X$. Define $f: X \rightarrow X$ by $f x=w$ for all $X \in X$. Then $f s x=w=s w=s f x$ for all $x \in X$, that is, $f \in C_{s}$. Clearly, (2.11) holds.

On the other hand, suppose that there exists a continuous self mapping $f$ of $X$ such that $f \in C_{s}$ and (2.11) holds. Let $A=\cap_{n \in \omega} S^{n} X$. From Lemma 1.1, we infer that $A$ is compact and $s A=A$. Since $f$ is continuous and $s \in C_{f}$, we have

$$
f A=f \cap_{n \in \omega} s^{n} X \subseteq \cap_{n \in \omega} s^{n} f X \subseteq \cap_{n \in \omega} s^{n} X=A=s A .
$$


Define the function $\phi(x)$ by $\phi(x)=F(s x, f x)$ for all $x \in A$. It is clear that $\phi(x)$ is continuous on $A$ and attains its minimum value at some $w \in A$. We claim that $s w=f w$. If not, from (2.12), there exists $p \in A$ satisfying $f w=s p$. Using (2.11), we conclude that

$$
\begin{aligned}
\phi(p)= & F(s p, f p)=F(f w, f p) \\
< & \max \left\{F(s w, s p), F(s w, f w), F(s p, f p), \frac{F(s w, f w) F(s p, f p)}{F(s w, s p)},\right. \\
& \left.\quad \frac{F(f w, f p) F(s w, f w)}{F(s w, s p)}, \frac{F(s w, f p) F(f w, s p)}{F(s w, s p)}\right\} \\
= & \max \{F(s w, f w), F(s p, f p)\} \\
= & F(s w, f w) \\
= & \phi(w)
\end{aligned}
$$

which is a contradiction to the choice of $w$. So $s w=f w$. By virtue of $f \in C_{s}$, we have

$$
f s w=s f w=s s w .
$$

Now suppose that $s s w \neq s w$. By (2.11) and (2.14), we get

$$
\begin{aligned}
F(s s w, s w)= & F(f s w, f w) \\
< & \max \left\{F(s s w, s w), F(s s w, f s w), F(s w, f w), \frac{F(s s w, f s w) F(s w, f w)}{F(s s w, s w)},\right. \\
& \left.\frac{F(f s w, f w) F(s s w, f s w)}{F(s s w, s w)}, \frac{F(s s w, f w) F(f s w, s w)}{F(s s w, s w)}\right\} \\
= & F(s s w, s w),
\end{aligned}
$$

which is a contradiction. Thus $s s w=s w$, that is, $s w$ is a fixed point of $s$. Therefore, the set $M$ of fixed points of $s$ is not empty. Now $s$ is continuous, so $M$ is closed. Since $M \subseteq A$ and $A$ is compact, $M$ is compact. Moreover, since $f$ and $s$ commute, $f(M) \subseteq M$. Note also that (2.11) restricted to $M$ reduces to (2.3). We can therefore apply Theorem 2.1(b4) to $f_{M}: M \rightarrow M$ to obtain a unique common fixed point $u$ of $f$ and $s$ in $M$. Since $M$ contains all the fixed points of $s, u$ is a unique common fixed point $f$ and $s$. This completes the proof.

THEOREM 2.3. Let $f, g$ be continuous compact self mappings of a metric space $(X, d)$ satisfying (2.1). Then $f$ and $g$ have a unique fixed point, respectively, and furthermore, for any $\alpha \in(0,1)$, there exist metrics $d^{\prime}$ and $d^{\prime \prime} \in M(X)$ relative to which $f$ and $g$ satisfy, respectively,

$$
d^{\prime}(f x, f y) \leq \alpha d^{\prime}(x, y), \quad d^{\prime \prime}(g x, g y) \leq \alpha d^{\prime \prime}(x, y),
$$

for all $x, y \in X$.

Proof. Let $A=\cap_{n \in \omega} f^{n} X, B=\cap_{n \in \omega} g^{n} X$, and $U=X$. As in the proof of Theorem 2.1, we have $A=B=\{w\}$. Lemma 1.1 ensures that (a5) and (a6) hold. Note that 
$d\left(f^{n} X\right), d\left(g^{n} X\right) \rightarrow 0$ as $n \rightarrow \infty$. Thus $f^{n} X$ and $g^{n} X$ squeeze into any neighborhood of $w$. That is (a7) is fulfilled. Thus Theorem 2.3 follows from Lemma 1.2. This completes the proof.

COROLLARY 2.4. Let $f$ be a continuous compact self mapping of a metric space $(X, d)$ satisfying

$$
F(f x, f y)<\delta\left(H_{f}(x), H_{f}(y)\right),
$$

for all $x, y \in X$ with $x \neq y$. Then $f$ has a unique fixed point.

Furthermore, for any $\alpha \in(0,1)$, there exists a metric $d^{\prime} \in M(X)$ relative to which $f$ satisfies

$$
d^{\prime}(f x, f y) \leq \alpha d^{\prime}(x, y)
$$

for all $x, y \in X$.

The following simple example reveals that Corollary 2.4 extends properly Theorem 1.1 of Janos [1] and Theorem 1 of Park [9].

EXAMPLE 2.5. Let $X=\{0,2,4,6,9\}$ with the usual metric. Define a mapping $f: X \rightarrow$ $X$ by $f 0=f 4=f 6=6, f 2=0$, and $f 9=2$. Then $f$ is a continuous compact self mapping of $X$. It is easy to check that

$$
d(f x, f y) \leq 6<9=\delta\left(H_{f}(x), H_{f}(y)\right),
$$

for all $x, y \in X$ with $x \neq y$. So the conditions of Corollary 2.4 are satisfied. But Theorem 1.1 of Janos [1] and Theorem 1 of Park [9] are not applicable since

$$
\begin{aligned}
& d(f 2, f 4)=6>2=\frac{1}{2}[d(2, f 2)+d(4, f 4)], \\
& d(f 2, f 4)=6=\delta(O(2,4, f)) .
\end{aligned}
$$

ACKNOWLEDGEMENT. The authors express their thank to the referee for his helpful suggestions, and the third author was supported by Korea Research Foundation Grant (KRF-99-005-D00003).

\section{REFERENCES}

[1] L. Janos, On mappings contractive in the sense of Kannan, Proc. Amer. Math. Soc. 61 (1976), no. 1, 171-175. MR 54\#13886. Zbl 342.54024.

[2] G. Jungck, Commuting mappings and fixed points, Amer. Math. Monthly 83 (1976), no. 4, 261-263. MR 53\#4031. Zbl 321.54025.

[3] M. S. Khan and B. Fisher, Some fixed point theorems for commuting mappings, Math. Nachr. 106 (1982), 323-326. MR 83k:54053. Zbl 501.54031.

[4] S. Leader, Uniformly contractive fixed points in compact metric spaces, Proc. Amer. Math. Soc. 86 (1982), no. 1, 153-158. MR 83j:54041. Zbl 507.54040.

[5] Z. Liu, Families of mappings and fixed points, Publ. Math. Debrecen 47 (1995), no. 1-2, 161-166. CMP 1362 279. Zbl 854.54039.

[6] _ On compact mappings of metric spaces, Indian J. Math. 37 (1995), no. 1, 31-36. CMP 1366 982. Zbl 839.54031.

[7] P. R. Meyers, A converse to Banach's contraction theorem, J. Res. Nat. Bur. Standards Sect. B 71B (1967), 73-76. MR 36\#4521. Zbl 161.19803. 
[8] S. Park, Fixed points of $f$-contractive maps, Rocky Mountain J. Math. 8 (1978), no. 4, 743750. MR 80d:54059. Zbl 398.54030.

[9]___ On general contractive-type conditions, J. Korean Math. Soc. 17 (1980), no. 1, 131140. MR 82e:54055. Zbl 448.54048.

Zeqing LiU: Department of Mathematics, LiaOning Normal University, Dalian, LIAONING 116029, CHINA

E-mail address: zeqingliu@sina.com.cn

Lili Zhang: Department of Mathematics, LiaOning Normal University, Dalian, LIAONING 116029, CHINA

Shin Min Kang: Department of Mathematics, GyeOngSAng NATional University, CHINJU 660-701, KOREA

E-mail address: smkang@nongae.gsnu.ac.kr 


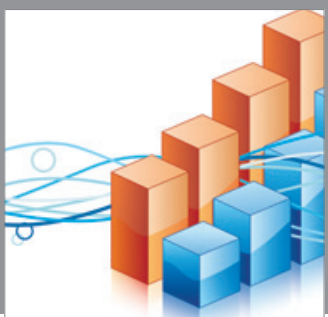

Advances in

Operations Research

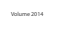

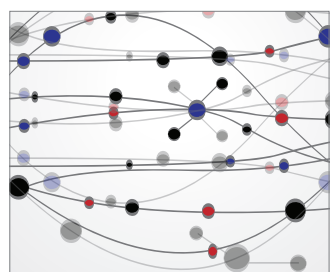

\section{The Scientific} World Journal
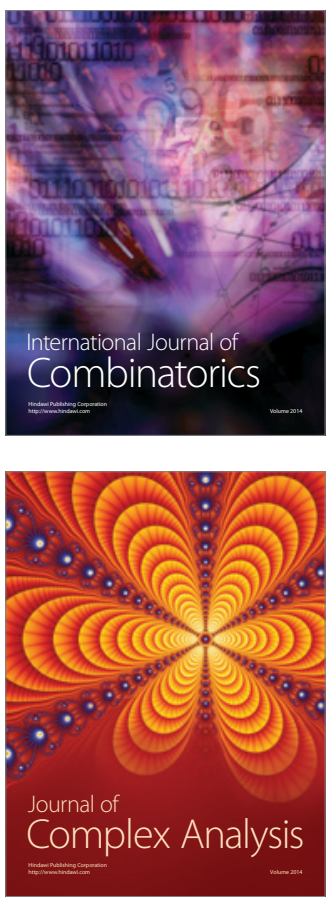

International Journal of

Mathematics and

Mathematical

Sciences
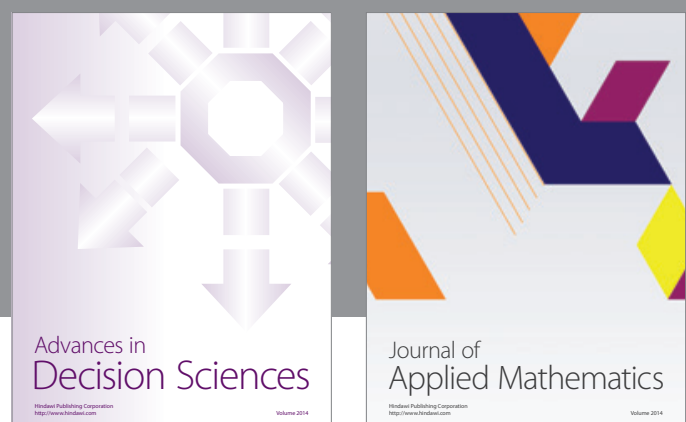

Journal of

Applied Mathematics
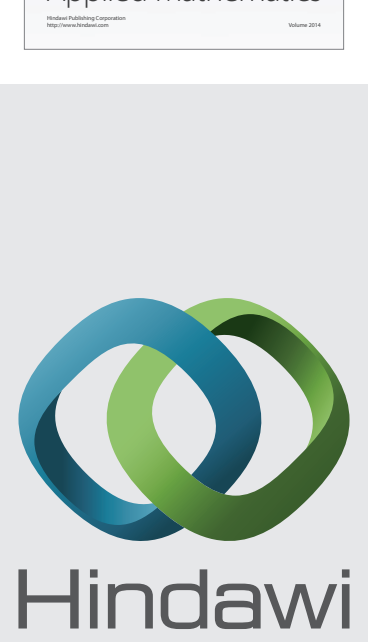

Submit your manuscripts at http://www.hindawi.com
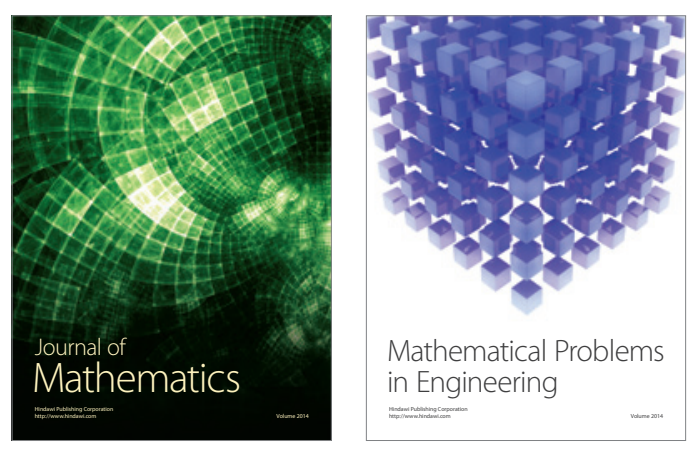

Mathematical Problems in Engineering
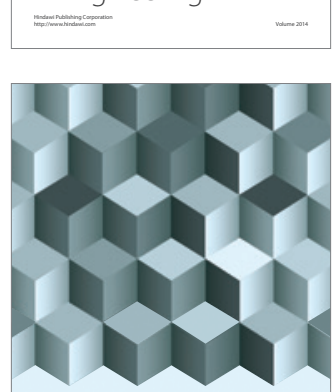

Journal of

Function Spaces
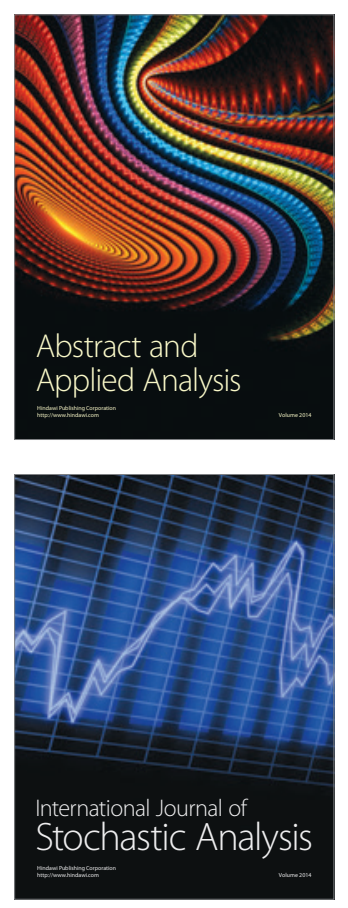

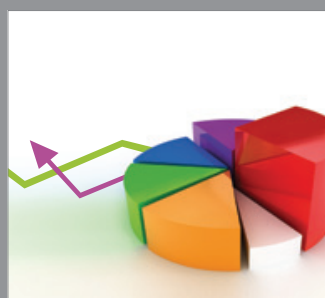

ournal of

Probability and Statistics

Promensencen
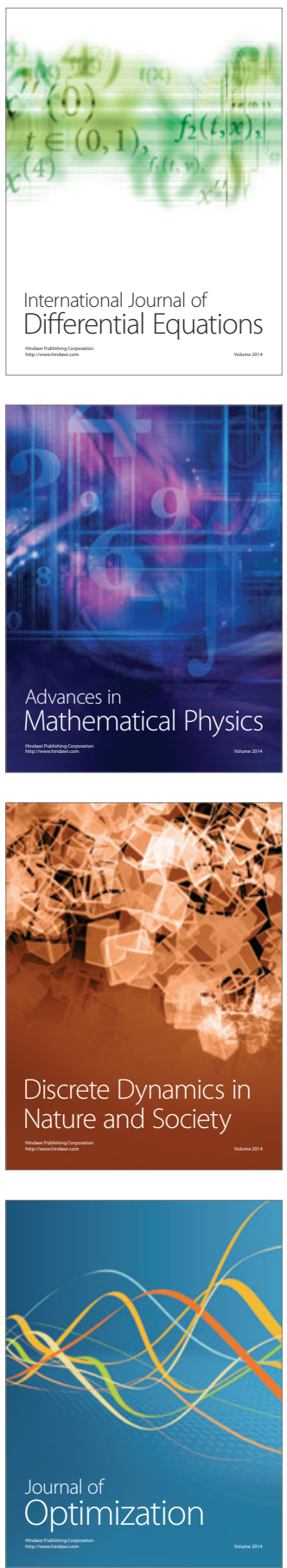